\title{
L'ARCHITECTURE MORPHO-SÉDIMENTAIRE DE LA BASSE PLAINE DE LA GARONNE MOYENNE EN AVAL DE TOULOUSE : PREMIERS RÉSULTATS
}

\author{
Jean-Michel CAROZZA ${ }^{1,2}$, Philippe VALETTE², Laurent CAROZZA², \\ Muriel LLUBES ${ }^{3}$, Laurie FERDINAND ${ }^{2}$, Serge EDOU OBAME ${ }^{3}$ \\ \& Laurent SÉVĖGNES ${ }^{4}$
}

\begin{abstract}
RÉSUMÉ
La moyenne Garonne, entre Toulouse et la confluence du Tarn, correspond à la zone d'élargissement de la basse plaine alluviale inondable, jusqu'à plus de $4 \mathrm{~km}$. Peu de travaux ont été menés sur l'architecture sédimentaire et la chronologie de la mise en place de cette unité géomorphologique. Dans ce travail, nous montrons que l'édification de la plaine en aval de Toulouse s'est effectuée en deux temps. Tout d'abord, une phase d'apports sédimentaires réduits, correspondant aux « limons jaunes », dont la mise en place s'effectue durant la phase alluviale pléniglaciaire entre 16000 ans cal. BP et au plus tard 8900 ans cal. BP. Cet épisode est encore très mal documenté. Une phase d'incision majeure remanie et érode les limons jaunes entre 8900 et 5000-4500 ans cal. BP. Cet épisode pourrait être contrôlé par le climat et la biostabilisation de la plaine en réponse à la reconquête par la chênaie mixte. Les accumulations de charbons alluviaux vers 4400 ans cal. BP pourraient signer les premiers impacts anthropiques majeurs sur la plaine, en relation avec les sites de Fontréal et Cassagna. Ces impacts anthropiques pourraient avoir favorisé la reprise de l'alluvionnement notamment sur l'effluent Hers-Girou à la confluence avec la Garonne. Après 4000 ans cal. BP, trois phases de mise en place des limons bruns de couverture sont identifiées vers 3700-3400 ans cal. BP, 2500-2000 BP et entre la fin du Moyen-Age et de la période Moderne.
\end{abstract}

Mots-clés : Garonne, Holocène, Tardiglaciaire, plaine alluviale, limons de couverture, géoarchéologie

\section{ABSTRACT}

THE MORPHO-SEDIMENTARY ARCHITECTURE OF THE LOWER MIDDLE GARONNE FLOODPLAIN DOWNSTREAM OF TOULOUSE: FIRST RESULTS

Between Toulouse and the Tarn confluence, the lower floodplain of the Middle Garonne widens and reaches more than $4 \mathrm{~km}$ wide. Both sedimentary architecture and chonology of its building are poorly documented by previous works. In this paper, we highlight that the floodplain, downstream of Toulouse, was mainly built in two episodes. First, an alluvial phase associated with reduced sediment supply, i.e. the " yellow silt », occurred and extended during the Pleniglacial alluvial phase $(16.0-8.9$ cal. ky BP). This episode remains poorly constrained. Downcutting and erosion partially removed the yellow silt during the 8.9 to $5.0-4.5$ cal. ky BP interval. Climate and biostabilisation by riparian forest (mixed oak forest) might control this episode. Charcoal accumulation in alluvial levels could be interpreted as the first human impact on riparian forest around $4.4 \mathrm{cal}$. ky BP. The local sites of Fontréal and Cassagna localized on the Pleistocene terrace are contemporary of this episode. Later, aggradation resumed on the Hers-Girou effluent at the Garonne confluence. After 4,000 cal. yr BP, we identified three deposition phases of the brown cover silts around 3,700-3,400 cal. yr BP, 2,500-2,000 BP and during Late Middle Age and the Modern period.

Keywords: Garonne, Holocene, Late Glacial, alluvial plain, cover silt, geoarchaeology

\section{1 - INTRODUCTION}

Au cours des 25 dernières années, les basses plaines alluviales des grands cours d'eau français ont fait l'objet d'intenses travaux qui ont contribué au renouvellement de la connaissance à la fois des processus d'édification et des facteurs de contrôle de leur architecture. En particulier, la prise en compte des données archéologiques au travers du développement de l'archéologie préventive a conduit à une intégration des approches stratigraphiques, géomorphologiques et géoarchéologiques. La vallée de la Garonne est restée relativement en marge de cette

\footnotetext{
${ }^{1}$ Université de Strasbourg, 3 rue de l'Argonne, FR-67000 STRASBOURG. Courriel : carozza@unistra.fr

${ }^{2}$ GEOgraphie De l'Environnement, UMR-5602 CNRS/UTM, 5 allées Antonio Machado, FR-31000 TOULOUSE. Courriels : valette@univtlse2.fr, laurent.carozza@univ-tlse2.fr, laurie,ferdinand@etu.univ-tlse2.fr

${ }^{3}$ GET - UMR 5563 CNRS-UPS, 14 avenue Édouard Belin, FR-31400 TOULOUSE. Courriel : muriel.1lubes@legos.obs-mp.fr

${ }^{4}$ Service Régional de l'Archéologie de Midi-Pyrénées - Ministère de la Culture, 32 rue de la Dalbade, FR-31000 TOULOUSE. Courriel :

laurent.sevegne@culture.gouv.fr
} 
dynamique de recherche et les données disponibles pour l'étude des relations entre les sociétés du passé et cet hydrosystème restent encore peu nombreuses, en particulier pour l'Holocène. Cette situation est d'autant plus paradoxale que ce terrain a constitué un pôle important de la recherche quaternaire dans les années 1970 (voir notamment Taillefer, 1951 ; Cavaillé, 1965 ; Icole, 1973 ; Hubschman, 1974). Plus récemment encore, les travaux de Lambert (1989) ont été à la base de la mise au point de la méthode hydro-géomorphologique qui s'est appuyée sur une cartographie précise et exhaustive des unités fonctionnelles, mais sans recourir sur un découpage chrono-stratigraphique de la plaine.

La basse plaine de la Garonne a longtemps été considérée comme une formation post-glaciaire (Enjalbert, 1960 ; Cavaillé, 1969) jusqu'aux travaux de Hubschman (1972, 1974). Sur la base de données paléo-pédologiques et de l'étude exoscopique des quartz d'une part et de la datation des formations loessiques notamment à Pompertuzat d'autre part $(20900 \pm 570 \mathrm{BP})$, Hubschman a attribué un âge « würmien » à l'essentiel de la basse plaine de la Garonne moyenne, notamment aux limons supérieurs, à l'exception des zones les plus près du fleuve. Lambert (1989) a partiellement repris cette interprétation pour le secteur immédiatement en aval de Toulouse, en suggérant un croisement entre les terrasses « würmienne » et la basse plaine récente, à savoir Holocène du fait de la pente plus faible de cette dernière. Bourgeat et al. (1984) suggèrent quant à eux, principalement sur la base de bois subfossiles, une progradation des formations de la plaine qui serait plus vieille en amont qu'en aval.

Récemment, Bruxelles et al. (2010) ont proposé une série de datations de la couverture limoneuse de la basse plaine au niveau de Castelnau-d'Estrétefonds. Pour ces auteurs cette couverture serait polygénique et résulte d'une mise en place entre le Pléniglaciaire terminal et la période Moderne. Ce schéma s'appuie sur des dates radiocarbones obtenues sur les « limons jaunes », un ensemble de faciès alluviaux à palustres décrits par Hubschman (1974), qui recouvrent les faciès grossiers de chenaux à la base de la séquence et des datations archéologiques. Les datations radiocarbones, réalisées sur coquilles, s'échelonnent entre $14200 \pm 100$ et $12505 \pm 50 \mathrm{BP}$ pour la base de la séquence. Le haut est daté par des structures archéologiques au sommet, au Néolithique moyen. Après une phase de répit entre le Néolithique moyen / final et l'Antiquité, une ultime phase de mise en place interviendrait et régulariserait une topographie alluviale jusque-là plus contrastée par la mise en place d'une couverture limoneuse sombre, les limons « bruns ». Si un tel découpage semble valide en aval immédiat de Toulouse, ce schéma pourrait ne pas pouvoir être généralisé à l'ensemble de la plaine.

Des observations et travaux complémentaires ont été réalisés depuis deux ans dans le secteur de la Garonne moyenne, principalement autour de Castelnau-d'Estrétefonds. Ils visaient à préciser l'organisation géomorphologique, la chrono-stratigraphie et l'évolution des paysages riverains à la fois dans leur composante abiotique, biologique et anthropique. Les principaux résultats sont présentés ici et sont discutés dans le cadre d'un schéma général d'évolution de la basse plaine.

\section{2 - ZONE D'ÉTUDE}

La Garonne est le troisième fleuve atlantique français par la taille de son bassin versant (Bassin versant : $57000 \mathrm{~km}^{2}$ ) et le plus méridional. Elle collecte des eaux en provenance de trois zones géographiques distinctes : les Pyrénées au sud, le Massif Central à l'est et le Bassin aquitain. De ce fait, elle est soumise à des influences climatiques aussi bien atlantiques que méditerranéennes, qui lui confèrent un régime de crues complexes (Pardé, 1935). Les travaux sur la géomorphologie de la plaine sont peu nombreux (Harlé, 1895 ; Lambert, 1989 ; Steiger \& Corenblit, 2000) ou restent très locaux. A l'échelle de l'ensemble du linéaire fluvial, Lambert (1989) a proposé un découpage en six secteurs sur la base de son fonctionnement hydrologique et du rapport entre la morphologie de la plaine alluviale et l'encaissant. En amont de la confluence avec l'Ariège, la Garonne conserve une vallée relativement étroite et encaissée. Ce n'est qu'au niveau de Toulouse que la vallée s'élargit et que la dynamique de méandrage s'amorce. Cette section dite de la Garonne moyenne toulousaine (Valette, 2002) se poursuit jusqu'à la confluence avec le Tarn. Elle n'est soumise qu'aux seules influences des crues pyrénéennes. Ce segment d'environ $80 \mathrm{~km}$ de long fait l'objet du présent travail (fig. 1). Au-delà s'étend la Garonne moyenne aval, puis maritime.

Dans la section de la Garonne moyenne, la vallée présente un dispositif de terrasses étagées sur près de $200 \mathrm{~m}$ de dénivelé, répartis sur une bande d'environ $16 \mathrm{~km}$ de large qui constitue un vaste couloir dégagé de la molasse tertiaire (Cavaillé, 1965 ; Hubschman, 1974). Si les niveaux les plus élevés ne sont préservés que de façon relictuelle et laissent des doutes sur les raccordements géométriques possibles, les niveaux inférieurs montrent une meilleure continuité. La basse plaine qui constitue le dernier d'entre eux est large de 3,5 à $4 \mathrm{~km}$ en moyenne. Elle est délimitée aussi bien à l'est qu'à l'ouest par un talus de terrasse dont la hauteur varie entre 6 et $22 \mathrm{~m}$. D'un point de vue topographique, cette unité correspond à l'enveloppe de la crue de 1875 qui s'est écoulée de bord à bord. La pente moyenne de la section reste forte, proche de 1,2\%o. L'homogénéité topographique actuelle de ce secteur pourrait n'être qu'apparente puisque Lambert (1989) suggère que ce secteur en aval de Toulouse où la plaine alluviale s'élargit correspondrait au croisement des terrasses « würmienne » et de la basse plaine Holocène. Cette option n'a pas été retenue par Cavaillé (1965, 1969) pour la carte géologique. L'architecture interne de cette unité est très mal documentée. Les données issues de la BSS du BRGM indiquent une épaisseur de l'ordre de 5 à $8 \mathrm{~m}$ et opposent des faciès de base conglomératique et sableuses d'une puissance de 2 à $5 \mathrm{~m}$ aux faciès supérieurs sablo-limoneux. 


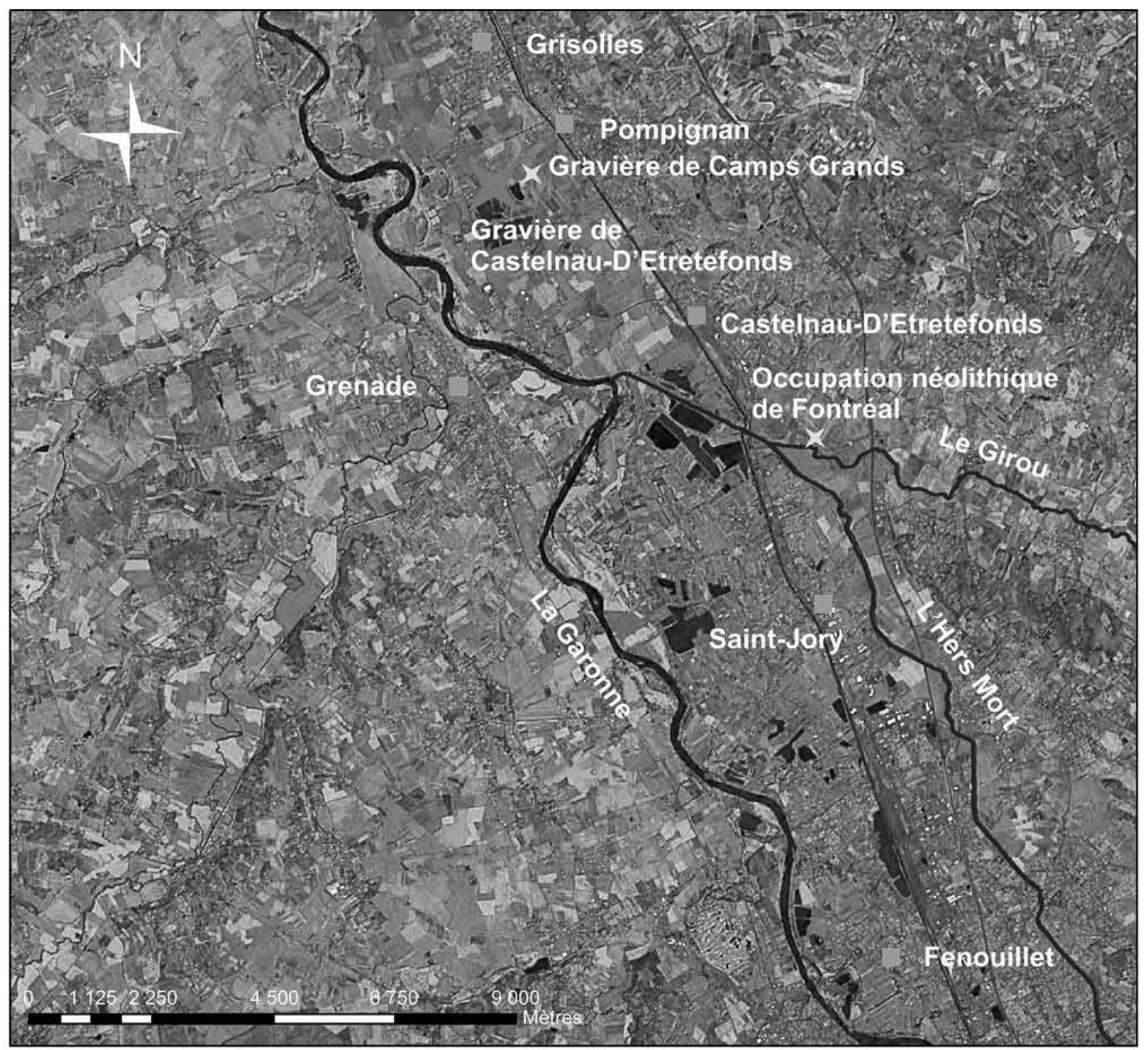

Fig. 1 : Carte de localisation de la zone d'étude.

Image satellite Landsat Circa 2000, composition colorée RVB. Réseau hydrographique d'après BD Carthage.

Fig. 1: Map of the study area. Remote sensing data from Landsat Circa 2000, RVB false color. Hydrographic network from Carthage Data Base.

\section{3 - LES DONNÉES}

Les données présentées ici ont été acquises par prospection systématique des gravières en activité dans le secteur de Castelnau-d'Estrétefonds (fig. 1). En complément, des profils de résistivité électrique ont été réalisés à l'aide d'un injecteur $\mathrm{ABEM}$ équipé de flûtes à 64 électrodes, suivant le protocole Schlumberger, afin de compléter ces données de terrain et tenter de restituer les géométries des corps sédimentaires de manière extensive et en profondeur. Sur les coupes, les faciès sédimentaires ont été décrits en suivant la méthodologie proposée par Miall (1996) pour les séries fluviales. De plus, des mesures de susceptibilité magnétique ont été réalisées in situ à l'aide d'un appareil SM 30. Les restes fauniques (principalement la malacofaune) et macro-charbons ont été échantillonnés sur place principalement pour datation. Les données archéologiques issues des diagnostics préalables à l'implantation des gravières et d'autres opérations voisines ont également été prises en compte. Enfin, un dépouillement exhaustif des données de la Base du Sous-Sol du BRGM a été réalisé. Ces données sont utilisées pour (1) modéliser la géométrie de la surface basale de ravinement correspondant aux plus bas niveaux d'érosion atteint par la Garonne et (2) pour contraindre les géométries des corps sédimentaires sur la base des grands faciès en complément des données acquises spécifiquement dans ce travail.

Deux sections ont fait l'objet d'un relevé et viennent compléter les données recueillies à proximité par Bruxelles et al. (2010) à Pompignan. La première (Castel 2) au niveau du front de taille actuel a permis l'observation suivant deux coupes perpendiculaires orientées NNE-SSO et NO-SE. La seconde (Castel 1), située sur une fosse d'extraction abandonnée permet de suivre un transect d'une cinquantaine de mètres de coupes en continu au SE des coupes précédentes. 


\section{1 - CASTELNAU-D’ESTRÉTEFONDS - ANCIEN FRONT}

La coupe Castel 2 (fig. 2) montre une séquence globalement grano-décroissante d'environ 4,00 m. Elle se décompose en quatre ensembles :

- US 1 : il s'agit d'une unité conglomératique grossière et hétérogène, généralement à stratifications planes obliques frustres ;

- US 2 : c'est une unité grossière à dominante sableuse à conglomératique à la base organisé en unité d'environs 18 à $20 \mathrm{~cm}$ qui montrent un tri granulométrique et un granoclassement normal. Les limites entre les unités sont légèrement obliques $(\mathrm{St})$. Les unités médianes sableuses montrent une base ravinante nette montrant une réactivation. On observe à $2,40 \mathrm{~m}$, des encroûtements racinaires en manchons ainsi qu'une carbonatation diffuse de la porosité qui atteste de processus bio-pédologiques et de battance de nappe. Les faciès de sables grossiers de la base renferment une très importante quantité de charbons dispersés dans la matrice mais faiblement roulés, ce qui atteste d'une origine proche. Trois charbons ont été prélevés pour datation radiocarbone à $-3,10,-2,80$ et $1,10 \mathrm{~m}$ de la surface ;

-US 3 : cette unité à dominante sableuse fine, de couleur grise est caractérisée par des stratifications planes, seul un niveau vers $1,80 \mathrm{~m}$ montrant une augmentation de la granulométrie et des structures sédimentaires ravinantes ou obliques. Ces niveaux sableux renferment une mala- cofaune conséquente vers 2,20 et 1,60 m. Les individus $\mathrm{du}$ premier niveau sont entiers et ne montrent pas de trace de transport, ce qui suggère une position primaire. Par contre, le niveau supérieur renferme des individus fragmentés et visiblement transportés et remaniés. Ils sont associés à des traces de carbonatation diffuse qui suggèrent un encroûtement par battance de nappe dans ce paléo-chenal en cours de colmatage rapide ;

- US 4 : cette unité limoneuse de couleur plus sombre et relativement homogène. Elle montre une structure prismatique à la base associée à des processus pédologiques humectation-dessication. Vers $0,50 \mathrm{~m}$, l'occurrence rare mais répétée de clastes d'un diamètre supérieur à $8 \mathrm{~cm}$ pourrait être relié à des apports anthropiques, ce niveau ayant livré des fragments de céramique protohistorique, peut-être du $2^{\mathrm{e}}$ Age du Fer. Cette unité est interprétée comme la phase ultime de colmatage d'un paléochenal déconnecté par des niveaux de débordement. Un charbon a été prélevé pour datation à $1,10 \mathrm{~m}$.

Ces données montrent une séquence de colmatage d'un paléochenal de la Garonne à charge sableuse dominante. L'inscription de la coupe au niveau d'une paléoforme fluviale méandriforme ainsi que l'existence de stratifications obliques larges de type point bar dans la partie nord de la coupe suggèrent que le chenal colmaté pourrait être de style à méandre à charge sableuse. La base de la séquence matérialise la phase de déconnection (US2) puis d'abandon progressif (US3). Au sommet de la séquence (US4) la paléoforme est totalement décon-

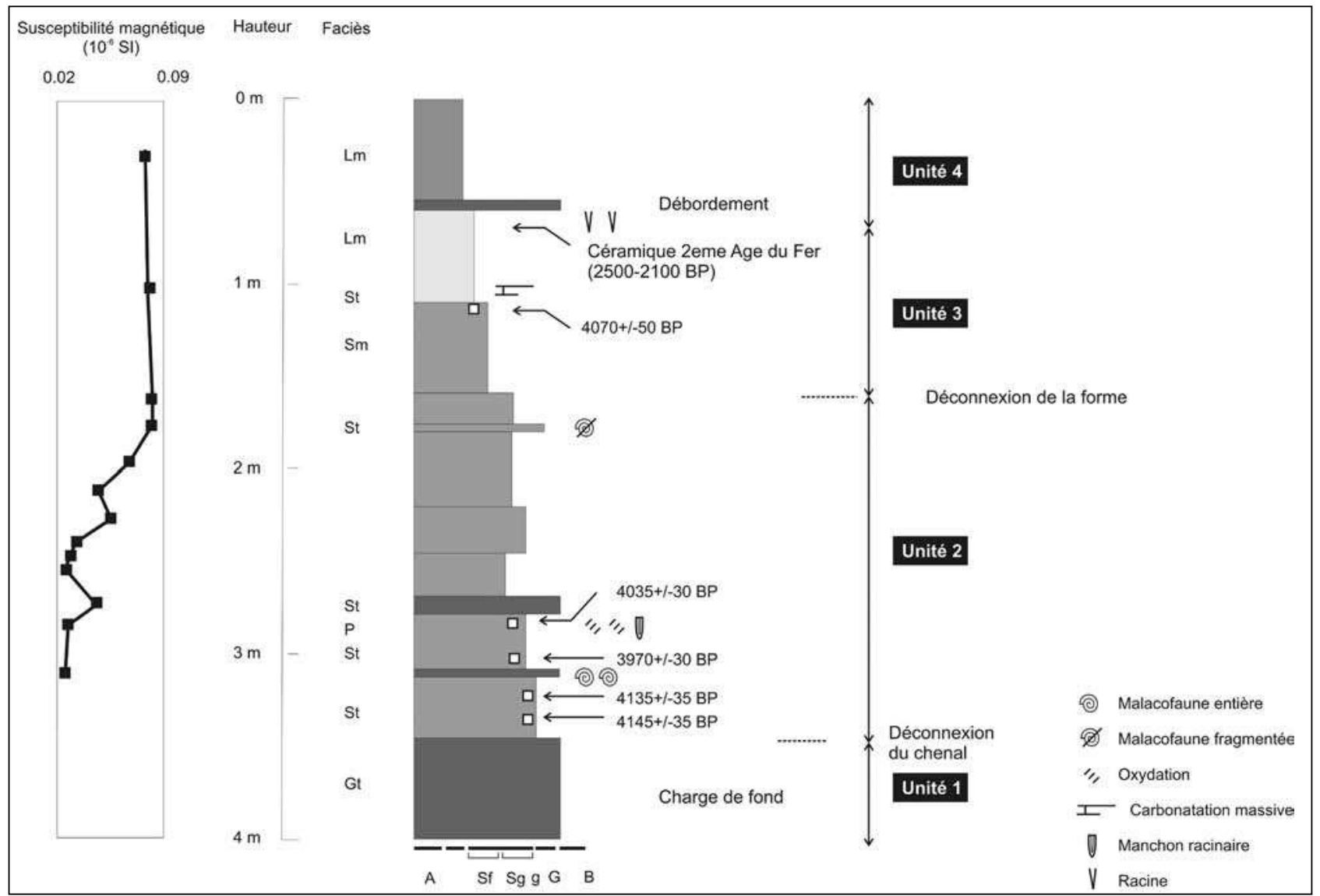

Fig. 2 : Profil transversal Castel 1 de la gravière de Castelnau-d'Estrétefonds (ancien front de taille).

Fig. 2: Castel 1 cross-section in Castelnau-d'Estrétefonds gravel pit (old excavation front). 
nectée et le colmatage se fait uniquement par débordement lors des crues. Les réactivations sont de moins en moins fréquentes vers le sommet et ont conduit à des remaniements de matériaux.

\section{2 - CASTELNAU-D'ESTRÉTEFONDS - NOUVEAU FRONT}

Les coupes de Castel 1 (fig. 3) montrent une organisation en trois ensembles distincts.

Le profil Castel 1-1 permet d'observer deux des US. Sa base est constituée par un limon sableux jaune barriolé. L'unité la plus profonde entre 2,10 et 1,60 m renferme de nombreuses coquilles, ainsi que des concrétions carbonatées en position secondaire. Latéralement, ce niveau peut passer à des formations plus grossières et légèrement chenalisées, parfois à faciès bioclastique. Dans ce dernier cas, des structures sédimentaires planes obliques peuvent être observées (Sbt). La partie supérieure de cet ensemble présente une teinte plus beige et une diminution des restes coquilliers. A 1,35 m un macrocharbon a été prélevé pour datation. A partir de $0,90 \mathrm{~m}$, une transition très franche mais non érosive sépare les limons jaunes d'un ensemble limono-argileux brun sombre à structure prismatique à la base et feuilletée au sommet. Ce dernier niveau renferme vers $0,40 \mathrm{~m}$ des galets et graviers épars à base planaire, reconnus sur l'ensemble des coupes.

La coupe Castel 1-2 montre au-dessus du faciès de limon jaune décrit précédemment, une US complexe de couleur brun clair qui ravine l'unité inférieure. La base de la séquence est un conglomérat à clastes jointifs de petite taille $(\Phi<2 \mathrm{~cm})$ dominés par des quartz laiteux et emballés dans une matrice sablo-limoneuse épais d'une dizaine de centimètres. Au-dessus, le niveau limono-sableux à structure prismatique indique une évolution pédogénétique. Un deuxième ensemble de limons légèrement sableux épais de $0,30 \mathrm{~m}$ et montrant la même évolution au sommet vient recouvrir cette unité. Le sommet de la séquence renferme un limon argileux sombre à nombreux fragments charbonneux qui a livré quelques fragments de céramique non roulés attribuée au Bronze Final. Une récurrence sableuse est également observée vers $0,35 \mathrm{~m}$ sous la surface.

Ces observations ont été complétées par la réalisation d'un profil électrique (fig. 4) avec dispositif à 64 électrodes écartées de 1,5 m. Le panneau couvre ainsi près de $100 \mathrm{~m}$ de long. Il montre la succession de trois unités principales. A la base, l'unité 1 à résistivité comprise entre 70 et $250 \Omega . \mathrm{m}^{-1}$ montre un sommet irré-

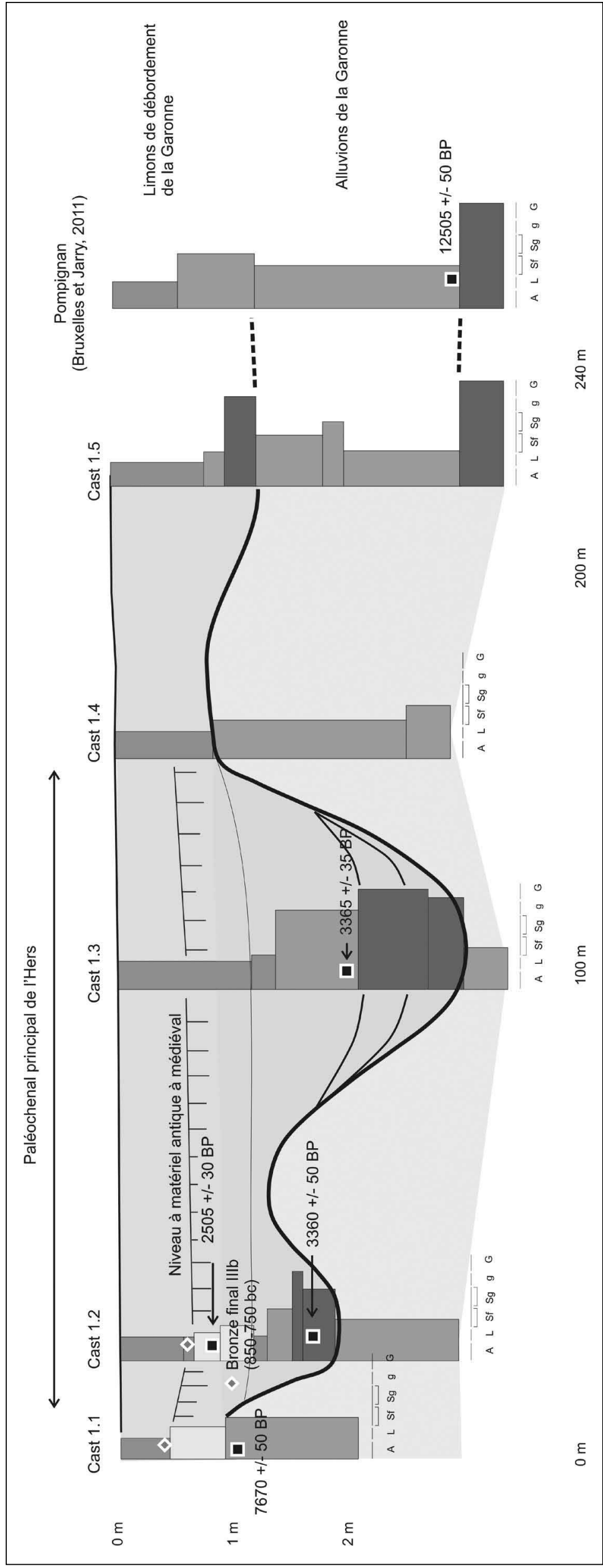




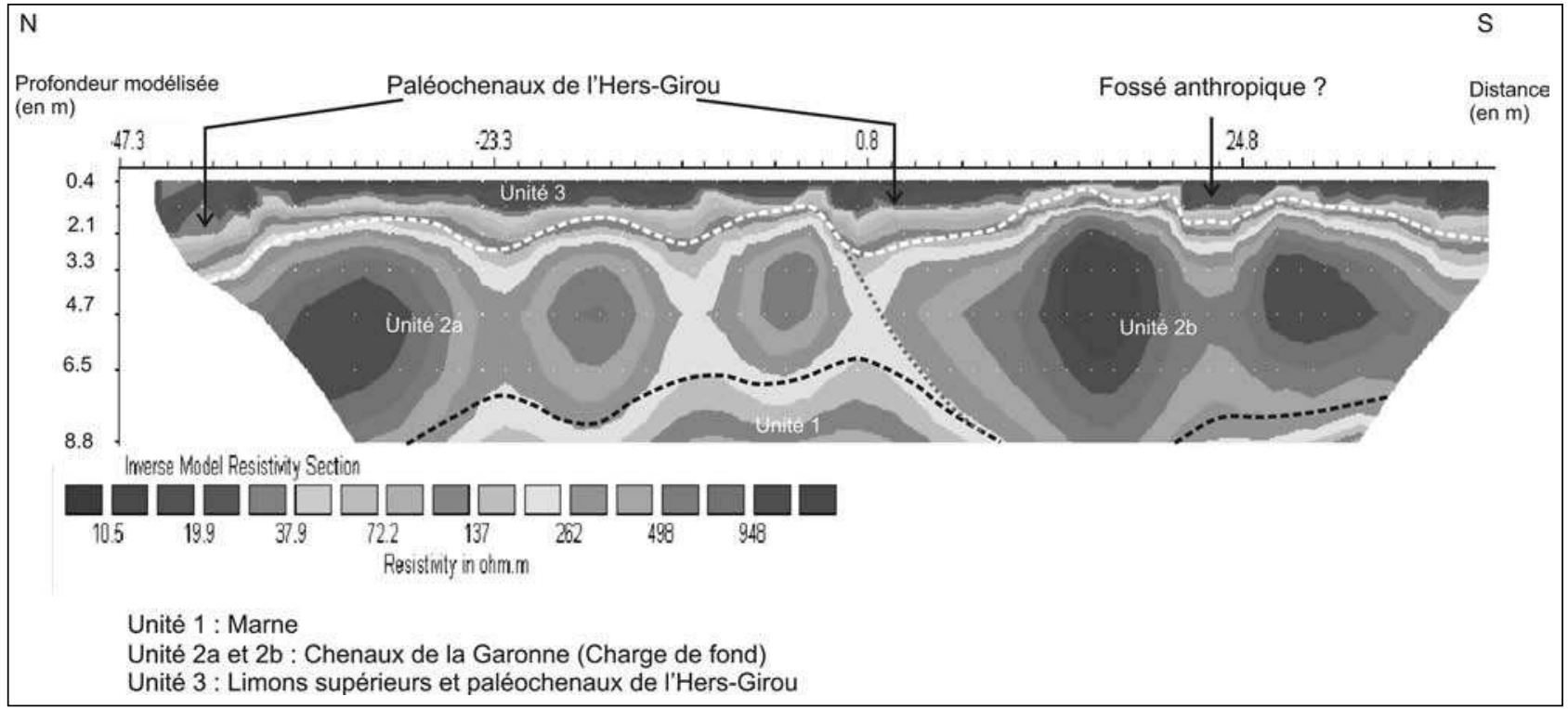

Fig. 4 : Profil électrique dans la gravière de Castelnau-d'Estrétefonds.

Fig. 4: Resistivity pseudo-section in Castelnau-d'Etrétefonds gravel pit.

gulier qui varie entre 6,5 et plus de $9 \mathrm{~m}$ de profondeur. Cette unité est ravinée par l'unité 2 à résistivité supérieure à $500 \Omega \cdot \mathrm{m}^{-1}$. Cette unité se décompose en deux motifs identiques (unités $2 \mathrm{a}$ et $\mathrm{b}$ ). Enfin, la séquence est surmontée par une unité à résistivité très faible, inférieure à $40 \Omega . \mathrm{m}^{-1}$, épaisse de $2 \mathrm{~m}$ en moyenne, mais dont la base est très irrégulière.

\section{3 - INTERPRÉTATION}

La coupe Castel 1-1 montre un ensemble de faciès typiques des limons jaunes qui viennent recouvrir les faciès grossiers de base conglomératique de la Garonne. Les données de résistivité électrique ainsi que les données géotechniques indiquent que les faciès de charge de fond ont ici une épaisseur d'environs 6 à $9 \mathrm{~m}$. Ce niveau de graves présente une base et un sommet irrégulier. En particulier, on observe une zone de surcreusement très prononcée visible sur la carte en isobathes (fig. 5). Les faciès supérieurs, limons jaunes et limons sombres, ont une épaisseur variable de l'ordre de $2 \mathrm{~m}$ et dessinent nettement un système de chenaux faiblement incisés. Si les faciès de limons jaunes ont indubitablement une origine garonnaise, les faciès grossiers observés sur la coupe Castel 1-2 indiquent clairement un remaniement depuis les niveaux de molasse et de terrasse Pléistocène par un affluent de la Garonne, l'Hers-Girou. Outre les éléments de géométrie des chenaux, cette interprétation est également étayée par la différence de matrice des conglomérats qui montre une couleur rouge-ocre et une texture argileuse. Les valeurs de susceptibilité magnétique sont toujours supérieures à celles de la coupe Castel 2 et voisines de $0,250^{*} 10^{-6}$ SI. Les données de terrain aussi bien que les données électriques montrent que la plaine de l'Hers-Girou était parcourue par de petits chenaux faiblement incisés et très mobiles, associés à une morphologie à chenaux multiples. La contemporanéité d'au moins deux chenaux est attestée par les data- tions radiocarbone. Toutefois, malgré une charge de fond importante, il n'est pas certain que l'Hers-Girou ait connu au cours de cette période une morphologie en tresse, la morphologie des chenaux évoquant d'avantage un cours d'eau à chenal droit à légèrement méandriforme.

\section{4 - CHRONOLOGIE DES DÉPÔTS}

Quatre dates radiocarbone ont été réalisées sur les coupes du secteur 1 (coupes Castel 1-1, Castel 1-2 et Castel 1-3, tab. 1) et sont complétées par les données archéologiques (fig. 3). La datation d'un charbon prélevé dans l'horizon supérieur des "limons jaunes », sous la couverture limoneuse brune, a livré un âge jeune (7670 $\pm 50 \mathrm{BP}$, soit 8975-8547 ans cal. BP) qui sera discuté. La base des faciès de colmatage des chenaux de l'Hers-Girou a quant à elle livré deux dates cohérentes : $3365 \pm 35 \mathrm{BP}$ et $3360 \pm 50 \mathrm{BP}$, soit respectivement 3484-3694 et 3715-3466 ans cal. BP. Le sommet du remplissage peut quant à lui être attribué sur la base des données archéologiques, au Bronze final 3 b ou au début de l'Age du Fer, soit une fourchette chronologique comprise entre 2800-2 675 ans cal. BP, là aussi en cohérence avec les datations radiocarbone. Une dernière date obtenue sur le niveau au-dessus des artefacts protohistoriques a livré une date $2505 \pm 30$, soit 2734-2471 ans cal. BP. Enfin, vers $0,70 \mathrm{~m}$ sous la surface, un niveau à matériel diffus a livré plusieurs fragments d'amphore et de tegulae, qui donnent un terminus post quem à l'édification de ce niveau supérieur.

Sur la coupe Castel 2, cinq dates radiocarbones ont également été réalisées sur charbons (tab. 1). Bien que présentant des inversions, elles indiquent une chronologie comprise entre 4820 et 4300 ans cal. BP. Les quatre datations basales peuvent être considérées comme relativement cohérentes. Elles indiquent un début de déconnexion du chenal méandriforme vers $4800-4400$ ans cal. BP. La date supérieure est quant à elle, manifestement remaniée. 


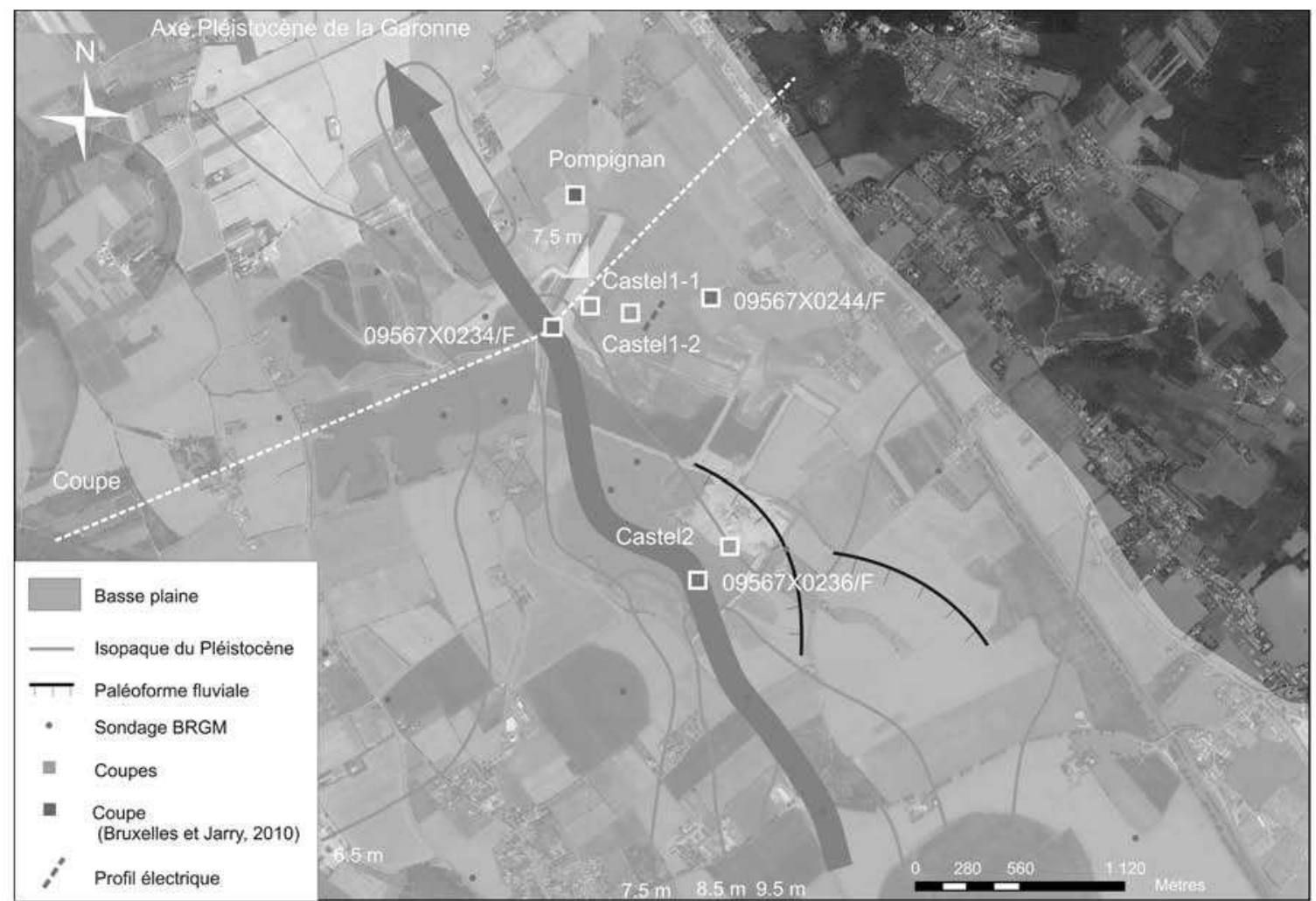

Fig. 5 : Carte isobathe du substratum marneux tertiaire aux environs de Castelnau-d'Estrétefonds. Image BD ORTHO IGN (2010; http://professionnels.ign.fr/bdortho).

Fig. 5: Isobath map of Tertiary marly bedrock around Castelnau-d'Etrétefonds. Image from IGN BD ORTHO database (2010; http://professionnels.ign. fr/bdortho).

La prise en compte des faits archéologiques (céramique du $2^{\mathrm{e}}$ Age du Fer in situ) semble plutôt indiquer une fin de colmatage de la paléoforme vers 2500 ans cal. BP, soit une date contemporaine de la date obtenue sur les limons bruns.

\section{4 - DISCUSSION}

Les données recueillies dans les gravières de Castelnaud'Estrétefonds viennent compléter les données de
Bruxelles et al. (2010) sur le site de Fontréal et de Lelouvier et Bruxelles (2008) sur Pompignan (fig. 5). Nos travaux permettent de discuter de la mise en place des « limons jaunes » tant d'un point de vue des milieux de dépôt que de la chronologie, de l'évolution de la plaine vers $4800 \mathrm{BP}$ et de la mise en place de la couverture limoneuse « brune ».

Les faciès sédimentaires des « limons jaunes » attestent d'une réduction des apports sédimentaires au cours du début du Tardiglaciaire, peut-être en relation avec les épisodes froids du Dryas 1 et 2 d'après les datations

\begin{tabular}{|c|c|c|c|c|}
\hline Type de matériaux & Code échantillon & $\mathbf{N}^{\circ}$ de laboratoire & Âge 14c & Age calibré à 2 $\boldsymbol{~}$ \\
\hline Charbon & CAST1-1 Ch1 & Poz-42111 & $7670 \pm 50 \mathrm{BP}$ & $8552-8385$ cal. BP \\
\hline Charbon & CAST2 Ch1 & Poz-42112 & $2505 \pm 30 \mathrm{BP}$ & 2734-2471 cal. BP \\
\hline Charbon & CAST2 Ch2 & Poz-42113 & $3360 \pm 50 \mathrm{BP}$ & 3715-3466 cal. BP \\
\hline Charbon & CAST3 Ch1 & Poz-42114 & $3365 \pm 35 \mathrm{BP}$ & 3692-3484 cal. BP \\
\hline Charbon & MGM3 -1.10 & Poz-42120 & $4070 \pm 50 \mathrm{BP}$ & 4812-4423 cal. BP \\
\hline Charbon & MGM3 -2.50 & Poz-42121 & $4035 \pm 30 \mathrm{BP}$ & $4778-4422$ cal. BP \\
\hline Charbon & MGM3 -2.80 & Poz-42122 & $3970 \pm 30 \mathrm{BP}$ & $4523-4299$ cal. BP \\
\hline Charbon & MGM3 -3.40 & Poz-42124 & $4145 \pm 35 \mathrm{BP}$ & $4826-4539$ cal. BP \\
\hline
\end{tabular}

Tab. 1 : Datations radiocarbone.

Tab. 1 : Radiocarbon datings. 
obtenues par Lelouvier et Bruxelles (2008) et Bruxelles et al. (2010). L'ambiance steppique des malacofaunes décrites par Bruxelles et al. (2010) s'accordent avec cette interprétation. Trochidea geyeri, Vallonia excentrica et Pupilla muscorum sont des espèces de milieux ouverts. Magnin (1993) indique pour Trochoidea geyeri, une gamme de températures comprises entre 4 et $12^{\circ}$. La datation obtenue sur un charbon de Quercus sp. au sommet de cette formation indique un âge beaucoup plus jeune compris entre 8552-8385 ans cal. BP. Nous l'interprétons comme un charbon de racine associé développement d'un sol sur les limons jaunes lors de la conquête par la chênaie mixte. La date obtenue est en effet très proche des datations les plus anciennes des bois fossiles découverts à Bourret (7380 \pm 40 , soit 8320-8070 ans cal. BP, Beta-282416) qui attestent de la biostabilisation puis de l'incision de cette formation au cours du début de l'Holocène. Cette formation n'est préservée qu'à l'est de la zone d'étude où elle porte plusieurs vestiges attribués au Néolithique et à l'Age du Bronze (Barbier et al., 1995 ; Tcheremissinoff et al., 2002). Les sites de cette période sont absents dans la partie ouest de la zone étudiée, ce qui est cohérent avec l'hypothèse d'un déstockage partiel des sédiments dans cette partie de la plaine. Cette phase de réduction des apports et/ou d'incision enregistrée par le contact entre les limons jaunes et les dépôts de l'HersGirou est clairement identifiée. Elle prend fin au plus tard vers 4 400-4300 ans cal. BP comme l'atteste le remplissage du chenal de Castel 1. Les premiers impacts anthropiques dans la plaine au cours du Néolithique moyen chasséen auraient ainsi pu se développer à la faveur de cette phase de réduction de l'activité fluviale. Avec la reprise de la sédimentation sur la marge de la plaine de la Garonne stricto sensu et la mise en place des formations de la confluence Hers-Girou sur la bordure nord de la plaine débute la phase de régularisation de la plaine et de ses marges. La mise en place de la couverture limoneuse " brune » semble ainsi se faire en trois temps comme l'indiquent les données acquises sur la coupe de Castel 2, vers 3 700-3 400 ans cal. BP, 2 500-2 000 BP puis après la période antique, peut-être durant le Petit Age Glaciaire (entendu comme la période couvrant du début du XIV ${ }^{\mathrm{e}}$ siècle au milieu du XIX ${ }^{e}$ siècle).

Un schéma d'évolution qui intègre l'ensemble des données stratigraphiques de terrain, les données archéologiques et les données géotechniques peut être proposé (fig. 6). Malgré son homogénéité topographique, la basse plaine se décompose en trois unités chrono-stratigraphiques distinctes qui reposent sur une discontinuité ravinant la molasse. Seules les deux unités principales du centre et de l'est de la plaine ont été étudiées pour l'instant.

La partie est de la plaine, entre le talus de terrasse et la N20, a préservé une unité principalement sableuse et limoneuse en surface (unité 3), alors qu'à l'ouest les formations sont plus grossières (unité 2). La mise en place de cette formation semble devoir être attribuée à l'extrême fin du Pléniglaciaire et au Tardiglaciaire entre 17,5 et 14,2 ka cal. BP, soit dès le début du Dryas ancien d'après les données déjà évoquées de Lelouvier et al. (2008) et Bruxelles et al. (2010). Le début de l'édification de la plaine pourrait être relié au désenglacement précoce du bassin versant qui serait déjà largement avancé dès avant 20000 BP (Pallas et al., 2006 ; Calvet et al., 2011 ; Delmas et al., 2012). Cette phase de construction reste à préciser tant d'un point de vue chronologique qu'environnemental, notamment en ce qui concerne un possible prolongement au cours du Dryas récent.

Dans la partie ouest, les formations observées sont très différentes, en accord avec les observations de Bruxelles (in Lelouvier \& Bruxelles, 2010). Ces auteurs interprètent cette opposition en termes de passage latéral de faciès entre les apports de la Garonne et le système Hers-Girou. La répartition des faciès sédimentaires entre apports de la Garonne et de l'Hers-Girou d'une part et la mise en séquence des données chrono-stratigraphiques que nous avons obtenues d'autre part ne permettent pas de valider cette hypothèse. Une transition érosive (incision) entre ces deux unités est plutôt à envisager. Le caractère local de celle-ci ne peut être envisagé du fait des datations obtenues sur la séquence de Castel 2 et de la largeur des formations observées. Cette phase d'incision est post-datée par le sol développé sur les limons jaunes (vers 8900 ans cal. BP) et antérieure au début du colmatage du chenal de Castel 1 (vers 4400 ans cal. BP). Cet épisode majeur de déstockage sédimentaire est observé dans d'autre systèmes fluviaux : entre 7400 et 5500 ans cal. BP dans la vallée du Rhône (Bravard, 1997 ; Berger, 2011), entre 7300 et 6400 ans cal. BP dans la Loire supérieure (Straffin \& Blum, 2002) et inférieure (Castanet, 2008), vers 6000 ans cal. BP dans la Seine (Lejeune et al., 2005) ou encore en Provence sur des systèmes de plus petite taille (Jorda et al., 2002). La relative ubiquité en Europe occidentale, (Dniepr : Kalicki \& Sanko, 1998 ; Danube hongrois : Pécsi \& Starkel, 1986) suggère une origine commune climatique ou bio-climatique. Le rôle respectif des impacts climatiques (RCC 6-5 et 4.2-3.8 notamment, Mayewski et al., 2004) et/ou des premiers impacts locaux anthropiques dans ce changement de dynamique reste incertain. Cet épisode est toutefois synchrone des premières occupations humaines néolithiques documentées dans la plaine de la Garonne au niveau de Fontréal (Barbier et al., 1995).

\section{5 - CONCLUSION}

Ces premiers travaux menés sur la partie amont de la Garonne moyenne tendent à indiquer que la mise en place de l'unité fonctionnelle de la basse plaine est principalement le résultat des dynamiques d'alluvionnement et d'incision survenues au cours du Tardiglaciaire et de l'Holocène. Deux phases majeures de dépôt peuvent ainsi être clairement identifiées. La première date du Tardiglaciaire et est associée à la mise en place des limons jaunes. Leur attribution aux phases froides Dryas 1 et 2 ainsi que leur relation aux formations grossières sousjacentes doivent être confirmées par de nouveaux travaux (datation et révision de la position stratigraphique des gisements de grandes faunes fossiles, travaux en cours). Durant la phase de biostabilisation associée à la mise 
Canal latéral

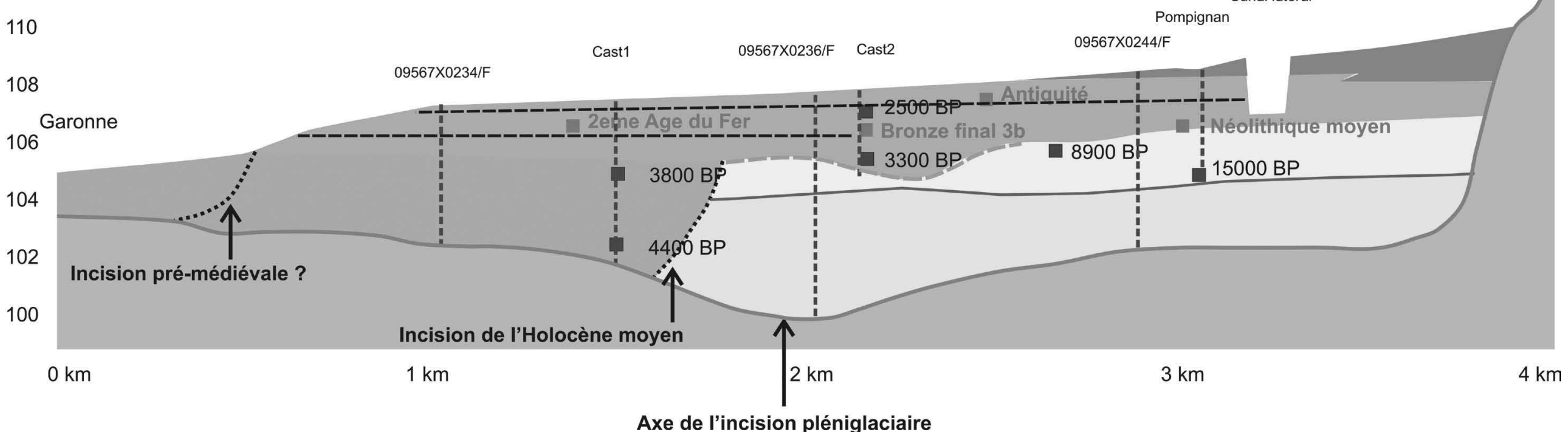

\section{Axe de l'incision pléniglaciaire} (30-18 ky BP ?)

\section{Marnes}

Graves Pléistocène supérieur

_ Incision pléniglaciaire

_ Contact grave - limons jaunes

- Ravinement de l'Hers-Girou

---_ Paléosols du 2eme holocène 
en place de la chênaie mixte, une phase d'incision se produit. Elle prend fin avant 4400 ans cal. BP avec un retour à une dynamique de déplacement des chenaux et au colmatage des paléochenaux de type méandriformes à charge sableuse. Les données recueillies sur le gisement de bois subfossiles de Bourret permettront de mieux cerner la chronologie et la nature de cet épisode et son caractère mono- ou polyphasé. La mise en place des limons bruns est polygénique et s'effectue en trois phases distinctes séparées par le développement de paléosols et la mise en place d'occupations humaines vers 3 700-3 400 ans cal. BP, 2 500-2000 BP et enfin entre le Bas Moyen-Age et la période Moderne.

\section{REMERCIEMENTS}

Les auteurs remercient les deux relecteurs, L. Bruxelles et $\mathrm{C}$. Castanet, pour leurs remarques constructives qui ont permis d'améliorer ce manuscrit. Les auteurs remercient MGM Sablières Réunies à Castelnau d'Estrétefonds pour les facilités d'accès au site d'exploitation.

\section{RÉFÉRENCES BIBLIOGRAPHIQUES}

BARBIER P., AMIEL C., BOUCHETTE A., CORDIER L. \& DEBERTONNE F., 1995 - ZAC Eurocentre (Castelnau-d'Estrétefonds). Document Final de Synthèse, Institut National de Recherches Archéologiques Préventives, Toulouse, $56 \mathrm{p}$

BERGER J.-F., 2011 - Hydrological and post-depositional impacts on the distribution of Holocene archaeological sites: The case of the Holocene middle Rhône River basin, France. Geomorphology, 129 (3-4), 126-182.

BOURGEAT F., ICOLE M. \& REVEL J.-C., 1984 - Les terrasses alluviales dans les Petites-Pyrénées et l'avant-pays molassique : les conditions de leur mise en place. Bulletin de l'Association Française pour l'Etude du Quaternaire, 21 (1-3), 60-66.

BRAVARD J.-P., VEROT-BOURRELY A. \& FRANC O., 1997 - Paléodynamique du site fluvial de Lyon depuis le Tardiglaciaire. In J.P. Bravard \& M. Prestreau (coords.), Dynamique du paysage : entretiens de géoarchéologie : Table ronde tenue à Lyon les 17 et 18 novembre 1995. Documents d'Archéologie en RhôneAlpes, 15. Lyon, 177-201.

BRUXELles L., PONS F., MAGNIN F. \& BERTRAND A. 2010 - Ages et modalités de la mise en place de la couverture limoneuse de la basse plaine de la Garonne d'après l'exemple du site de Fontréal (Castelnau-d'Etrétefonds, Haute Garonne). Quaternaire, 21 (4), 475-484.

CALVET M., DELMAS M., GUNNELL Y., BRAUCHER R. \& BOURLĖS D., 2011 - Recent advances in research on Quaternary glaciations in the Pyrenees. In J. Ehlers, P.L. Gibbard \& P.D. Hugues (eds.), Quaternary Glaciations, extent and chronology: A closer look. Developments in Quaternary Science, 15. Elsevier, Amsterdam, 127-139.

CASTANET C., 2008 - La Loire en val d'Orléans : dynamiques fluviales et socio-environnementales durant les derniers 30000 ans : de l'hydrosystème à l'anthroposystème. Thèse de Doctorat, Université Paris 1 Panthéon-Sorbonne, Paris, $545 \mathrm{p}$.

CAVAILLÉ A., 1965 - Les unités morphologiques des basses plaines de la Garonne. Revue Géographique des Pyrénées et du Sud-Ouest, 36 (3), 243-278.

CAVAILLÉ A., 1969 - Les terrasses de la Garonne à l'Ouest de Toulouse. In F. Taillefer (dir.), Livret-guide de l'excursion A6 : Pyrénées-Orientales et Centrales, Roussillon, Languedoc occidental. VIII Congrès INQUA, Paris, 1969. Imprimerie Biscaye Frères, Bordeaux, 16-23

DELMAS M., CALVET M., GUNNELL Y., BRAUCHER R. \& BOURLÈS D., 2012 - Les glaciations quaternaires dans les Pyrénées ariègeoises : approche historiographique, données paléogéographiques et chronologiques nouvelles. Quaternaire, 23 (1), 61-85.
ENJALBERT H., 1960 - Le Pays Aquitain. Le modelé et les sols. Thèse de Doctorat, Université de Paris, Paris. Imprimerie Bière, Bordeaux, $618 \mathrm{p}$.

HARLÉ É., 1895 - Observations sur les alluvions de la Garonne dans le secteur de Toulouse. Bulletin de la Société Géologique de France, $3^{\mathrm{e}}$ série, 23, 490-503.

HUBSCHMAN J., 1972 - L'âge de la basse plaine garonnaise des Pyrénées au confluent du Tarn. Comptes Rendus Hebdomadaires des Séances de l'Académie des Sciences. Série D, Sciences naturelles, 275, 651-653.

HUBSCHMAN J., 1974 - Morphogenèse et pédogenèse quaternaires dans le piémont des Pyrénées garonnaises et ariégeoises. Thèse de Doctorat, Université de Toulouse-Le Mirail, Toulouse, 745 p.

ICOLE M., 1973 - Géochimie des altérations dans les nappes d'alluvions du piémont occidental nord-pyrénéen : essai de paléopédologie quaternaire. Thèse de Doctorat d'Etat, Université Paris 6 Pierre et Marie Curie, Paris, 328 p.

KALICKI T. \& SANKO A.F., 1998 - Palaeohydrological changes in the Upper Dneper Valley, Belarus during the last 2000 yrs. In G. Benito, V.R. Baker \& K.J. Gregory (eds.), Palaeohydrology and environmental change. Wiley, Chichester \& New York, 125-138.

LAMBERT R., 1989 - Atlas hydraulique de la Garonne : du Pont du Roy au Bec d'Ambès. Tome 2, Schéma de protection contre les eaux de la Garonne. Syndicat Mixte d'Etude et de Programmation pour l'Aménagement de la Garonne, Toulouse, 46 planches couleurs au $1 / 25000^{\mathrm{e}}$

LEJEUNE Y., PASTRE J.-F, ARCAY D., AUBRY C., CAMERLYNCK J., CONFALIONERI Y., LANCHON Y., VERGNAUD D. \& VISSAC C., 2005 - Variabilité de l'enregistrement alluvial holocène ; l'exemple du méandre de la Haute-Île. Quaternaire, 16 (4), 299-314.

LELOUVIER L.-A. \& BRUXELLES L., 2008 - Le diagnostic archéologique de Pompignan (Haute Garonne). Document Final de Synthèse, Institut National de Recherches Archéologiques Préventives, Toulouse, $64 \mathrm{p}$.

MAGNIN F., 1993 - Trochoidea geyeri (Soós, 1926) (Pulmonata, Helicidae) in south-eastern France: ecology, biogeography and Quaternary history. Scripta Geologica. Special Issues, 2, 291-295.

MAYEWSKI P.A., ROHLING E.E., STAGER J.C., KARLÉN W., MAASCH K.A., MEEKER L.D., MEYERSON E.A., GASSE F., VAN KREVELD S., HOLMGREN K., LEE-THORP J., ROSQVIST G., RACK F., STAUBWASSER M., SCHNEIDER R.R. \& STEIG E.J, 2004 - Holocene climate variability. Quaternary Research, 62 (3), 243-255.

MIALL A., 1996 - The geology of fluvial deposits: sedimentary facies, basin analysis and petroleum geology. Springer-Verlag, Berlin, 582 p.

PALLAS R., RODÈS A., BRAUCHER R., CARCAILLET J., ORTUNO M., BORDONAU J., BOURLES D., VILAPLANA J.M., MASANA E. \& SANTANACH P., 2006 - Late Pleistocene and Holocene glaciation in the Pyrenees: a critical review and new evidence from ${ }^{10} \mathrm{Be}$ exposure ages, south-central Pyrenees. Quaternary Science Reviews, 25 (21-22), 2937-2963.

PARDÉ M., 1935 - Le régime de la Garonne. Revue Géographique des Pyrénées du Sud-Ouest, 6, 105-262.

PÉCSI M. \& STARKEL L. (eds.), 1986 - Paleogeography of the Carpathian regions: proceedings of the Polish-Hungarian Paleogeographical Seminar, Tata, Hungary, October 16-22, 1986. Theory, Methodology, Practice, 47. Hungarian Academy of Sciences, Budapest, $191 \mathrm{p}$.

STRAFFIN E.C. \& BLUM M.D., 2002 - Late and post-glacial fluvial dynamics of the Loire river, Burgundy, France. In J.-P. Bravard \& M. Magny (eds.), Les fleuves ont une histoire. Errance, Paris, 85-100.

STEIGER J. \& CORENBLIT D., 2000 - Les ajustements morphologiques contemporains du lit mineur de la Garonne (France) et leurs effets sur 1'hydrosystème fluvial. Zeitschrift fur Geomorphologie. Supplementband, 122, 227-246.

TAILLEFER F., 1951 - Le Piémont des Pyrénées françaises : contribution à l'étude des reliefs de Piémont. Thèse de Doctorat, Université de Toulouse, Toulouse. E. Privat, Toulouse, 383 p.

TCHEREMISSINOFF Y., MARSAC P., BERTHET A., JARRY M., LOISELIER L., MARTIN H. \& RANCHER C., 2002 - Cassagna 2. Occupation néolithique et protohistorique (Blagnac, Haute Garonne). Document Final de Synthèse, Institut National de Recherches Archéologiques Préventives, Toulouse, $122 \mathrm{p}$

VALETTE P., 2002 - Les paysages de la Garonne: les métamorphoses d'un fleuve : entre Toulouse et Castets-en-Dorthe. Thèse de Doctorat, Université de Toulouse Le Mirail, Toulouse, 554 p. 